\title{
Absolute frequency measurements of the molecular iodine hyperfine transitions at $535 \mathrm{~nm}$
}

\author{
Nang-Chian Shie, ${ }^{1}$ Shih-En Chen, ${ }^{2}$ Chun-Yu Chang, ${ }^{3}$ Wen-Feng Hsieh, ${ }^{1}$ and Jow-Tsong Shy ${ }^{2,3, *}$ \\ ${ }^{1}$ Department of Photonics and Institute of Electro-Optical Engineering, National Chiao Tung University, \\ Hsinchu 30050, Taiwan \\ ${ }^{2}$ Department of Physics, National Tsing Hua University, Hsinchu 30013, Taiwan \\ ${ }^{3}$ Institute of Photonics Technologies, National Tsing Hua University, Hsinchu 30013, Taiwan \\ *Corresponding author: shy@phys.nthu.edu.tw
}

Received May 8, 2013; accepted May 28, 2013;

posted June 4, 2013 (Doc. ID 190086); published June 28, 2013

\begin{abstract}
We report absolute frequency measurements of the $a_{1}, a_{10}$, and $a_{15}$ hyperfine components of molecular iodine $\mathrm{P}(28)$

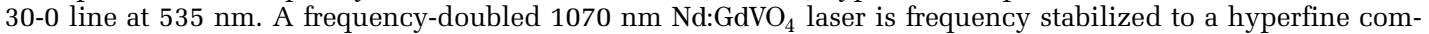
ponent of $\mathrm{I}_{2}$ using the third-harmonic demodulation technique. The frequency stability of $3 \times 10^{-12}$ is achieved at $10 \mathrm{~s}$ averaging time when its frequency is stabilized to the $a_{1}$ component. An optical frequency comb is used to measure its absolute frequency. The pressure shift is investigated to obtain the absolute frequency at zero pressure. The effect of pressure and power broadening of the $a_{10}$ component are also investigated. This light source can be used for investigating parity nonconservation effect in atomic thallium. (c) 2013 Optical Society of America OCIS codes: (300.6320) Spectroscopy, high-resolution; (300.6390) Spectroscopy, molecular; (300.6460)

Spectroscopy, saturation.

http://dx.doi.org/10.1364/JOSAB.30.002022
\end{abstract}

\section{INTRODUCTION}

The broad spectrum of molecular iodine has been widely used for optical wavelength reference or laser frequency stabilization in the visible and near-infrared region. The iodinestabilized lasers provide frequency references for various high-precision atomic experiments [1-5] and several of them are recommended as the wavelength standards by the Comité International des Poids et Mesures [6,7]. The extensive precision measurements of iodine spectrum allow us to characterize and calculate the iodine hyperfine structures [8]. The accuracy of the calculation depends on the high-precision transition frequencies measured by available stable laser sources. Diode-pumped solid-state lasers (DPSS) with good beam quality, narrow linewidth, and high stability are outstanding light sources for laser spectroscopy. Nonlinear optical-frequency conversion, such as second-harmonic generation, enables the infrared DPSS to be locked to the hyperfine transitions of molecular iodine in the visible. For example, the iodine-stabilized frequency-doubled Nd:YAG lasers at $532 \mathrm{~nm}$ show excellent stability and the absolute frequencies of iodine hyperfine transitions near $532 \mathrm{~nm}$ have been measured to very high accuracy using the optical frequency comb (OFC) [9-13].

In this report we present the Doppler-free saturation spectroscopy of the hyperfine transitions of ${ }^{127} \mathrm{I}_{2} \mathrm{P}(28)$ 30-0 line at $535 \mathrm{~nm}$ using a frequency-doubled $1070 \mathrm{~nm} \mathrm{Nd:GdVO}$ laser. Saturation spectroscopy is used to observe the hyperfine spectrum. The Nd:GdVO 4 laser is stabilized to the $a_{1}, a_{10}$, and $a_{15}$ hyperfine components and their absolute frequencies are measured by an OFC. The pressure shift is investigated to obtain the absolute frequency at zero pressure. The effect of pressure and power broadening of the $a_{10}$ component are also investigated. Our results are useful for improving the theoretical calculation. In addition, the frequency of this iodine line is very close to the $6 \mathrm{P}_{3 / 2} \rightarrow 7 \mathrm{~S}_{1 / 2}$ transition of the thallium atom. The parity nonconservation (PNC) effect has been observed in atomic thallium system using $6 \mathrm{P}_{1 / 2} \rightarrow 6 \mathrm{P}_{3 / 2}$ transition in 1995 [14,15]. Electromagnetically induced transparency (EIT) has been proposed to improve the PNC measurement [16] in which a $535 \mathrm{~nm}$ laser is required. Therefore, this laser is useful for searching the PNC effect in thallium.

\section{EXPERIMENTS}

The schematic diagram of our experiment is shown in Fig. 1. The laser source is a frequency-doubled $1070 \mathrm{~nm} \mathrm{Nd:GdVO}$ laser. The $1070 \mathrm{~nm} \mathrm{Nd:GdVO}$ laser uses a volume Bragg grating (VBG) as the output coupler and the wavelength selector. This $1070 \mathrm{~nm}$ laser is similar to our early version [17] except an $\mathrm{Nd}: \mathrm{GdVO}_{4}$ laser crystal with a spherical input surface [ $R=30 \mathrm{~cm}$, high-transmission (HT) at $808 \mathrm{~nm}$, highreflection (HR) at $1070 \mathrm{~nm})$ ] and a flat end surface [(antireflection $(\mathrm{AR})$ at 808 and $1070 \mathrm{~nm})$ ] is adopted to replace the concave cavity mirror and the flat-flat $\mathrm{Nd}$ : $\mathrm{GdVO}_{4}$ laser crystal to increase the frequency stability. The spherical surface of this laser crystal acts as the concave mirror of the plano-concave laser cavity. The laser crystal is mounted on a PZT for cavity length tuning. The laser is put in an aluminum housing whose temperature is tuned by a thermoelectric temperature controller to reduce the air and thermal fluctuations. The frequency of the laser can be tuned coarsely by the VBG temperature and finely by the PZT voltage. The VBG temperature is set to $45^{\circ} \mathrm{C}$ for the iodine $\mathrm{P}(28) 30-0$ line wavelength. There are three main output beams due to the reflections of the Bragg grating planes and the VBG surfaces. All outputs are 


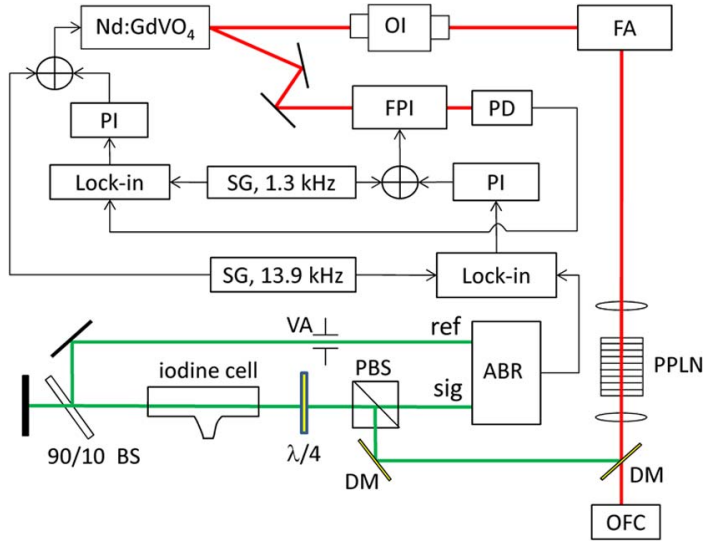

Fig. 1. Experimental layout of the iodine laser stabilization. OI, optical isolator; FA, fiber amplifier; FPI, Fabry-Perot interferometer; PD, photodiode; PI, PI servo loop; SG, signal generator; Lock-in, Lockin amplifier; VA, variable aperture; ABR, auto-balanced receiver; BS, beam splitter; $\lambda / 4$, quarter-wave plate; DM, dichroic mirror.

linearly polarized. The central beam is used to carry out our spectroscopy experiment. One of the side beams is directed into a confocal Fabry-Perot interferometer (FPI). The laser frequency is locked to the FPI to reduce its frequency jitter. To lock the laser to the FPI, the FPI cavity length is modulated at $1.3 \mathrm{kHz}$. The $1070 \mathrm{~nm}$ light signal after the FPI cavity is demodulated to obtain the error signal for locking. The power of the central beam is boosted by a $900 \mathrm{~mW} \mathrm{Yb-doped} \mathrm{fiber}$ amplifier. The amplified $1070 \mathrm{~nm}$ laser beam is then focused into a $50 \mathrm{~mm}$ long $\mathrm{MgO}$ doped periodically poled lithium niobate (MgO:PPLN) crystal with a lens $(f=100 \mathrm{~mm})$ for frequency doubling. The PPLN crystal is mounted in a temperature-stabilized oven and the quasi-phase matching condition is achieved around $107^{\circ} \mathrm{C}$. After passing through the PPLN, the frequency-doubled $535 \mathrm{~nm}$ light is collimated to $8.4 \mathrm{~mm}$ in diameter. The $1070 \mathrm{~nm}$ light is separated out by two dichroic mirrors (DMs). The $1070 \mathrm{~nm}$ light passing through the first DM is transferred to an OFC [18] by a polarization maintaining single-mode fiber for frequency measurement. The $535 \mathrm{~nm}$ green light power after these two DMs is typically $46 \mathrm{~mW}$ at an input IR power of $700 \mathrm{~mW}$, which corresponds to $6.5 \%$ power conversion efficiency.

The second-harmonic generated $535 \mathrm{~nm}$ light is then used to observe the saturation absorption signal of the hyperfine components of the $\mathrm{P}(28)$ 30-0 line in an iodine vapor cell. The linear polarized green light passing through a polarizing beam splitter (PBS) and a quarter-wave plate $(\lambda / 4)$ is transformed into circularly polarized light, and goes through an iodine vapor cell acting as the saturating beam. The $6 \mathrm{~cm}$ long iodine vapor cell with plane windows is slightly tilted in the beam direction to prevent backreflection into the laser system. The cold finger of the cell can be temperature controlled from 0 to $23^{\circ} \mathrm{C}$ and accordingly the iodine vapor pressure from 4.1 to $34.9 \mathrm{~Pa}$. The circular polarized green light is further separated by a $90 / 10$ beam splitter (BS). The weaker beam after the BS is retroreflected by a mirror into the iodine cell acting as the probe beam. Then it passes through the $\lambda / 4$ plate and PBS to the signal input of an autobalanced receiver (New Focus 2017 Nirvana). The stronger beam is directed into the reference input of the autobalanced receiver. The autobalanced receiver is used to suppress the intensity noise of the $535 \mathrm{~nm}$ light. The optimum ratio of signal and reference optical powers for noise cancellation is achieved by a variable aperture in front of the reference input. The signal monitor port of the receiver was used to observe the probe beam intensity. To obtain the third-derivative saturated absorption signal of the iodine $\mathrm{P}(28)$ 30-0 line for frequency locking the laser frequency is modulated at $13.9 \mathrm{kHz}$. The balanced signal from the receiver is demodulated with a lock-in amplifier (Standard Research Systems SR830) at the third-harmonic of $13.9 \mathrm{kHz}$. The time constant is set to $30 \mathrm{~ms}$ at $12 \mathrm{~dB} /$ oct. To lock the laser frequency to the hyperfine transition, the resulting third-derivative signal is feed through a proportional and integral (PI) servo loop to control the FPI cavity length.

Once the laser is locked to one of the hyperfine transitions, the $1070 \mathrm{~nm}$ laser frequency is measured by a self-referenced OFC [18], which is based on a $1 \mathrm{GHz}$ mode-locked Ti:sapphire laser with spectrum expanded using a microstructure fiber. The repetition frequency $f_{\text {rep }}$ and offset frequency $f_{\text {offset }}$ of our OFC are phase locked to a GPS-disciplined Rb clock. The accuracy of our OFC is better than $1 \times 10^{-12}$ at a $1000 \mathrm{~s}$ measurement time. The transition frequency is determined by the following relation:

$$
f_{\text {iodine }}=2 f_{I R}=2\left(N f_{\text {rep }} \pm f_{\text {offset }} \pm f_{\text {beat }}\right)
$$

where $N$ is a mode number and $f_{\text {beat }}$ is the beat frequency between laser and the nearest comb line. To determine $N$, the laser frequency is measured by a wavemeter with uncertainty less than $1 \mathrm{GHz}$. The absorption spectrum has also been scanned over a range of several gighertz to identify the transitions according to the pattern predicted by the IodineSpec5 [8]. The repetition rate is much larger than the expected uncertainty of IodineSpec5, so the mode number $N$ can be determined by comparing the resulting frequency value using Eq. (1) with the IodineSpec5 prediction. The frequency measurement is further confirmed using different repetition rate and offset frequency of the OFC.

\section{RESULTS AND DISCUSSION}

Figure 2 shows the saturation absorption spectrum of the $\mathrm{P}(28) 30$ - 0 line of ${ }^{127} \mathrm{I}_{2}$ at $535 \mathrm{~nm}$ using the third-harmonic demodulation method. Here, the $1070 \mathrm{~nm}$ laser is locked to

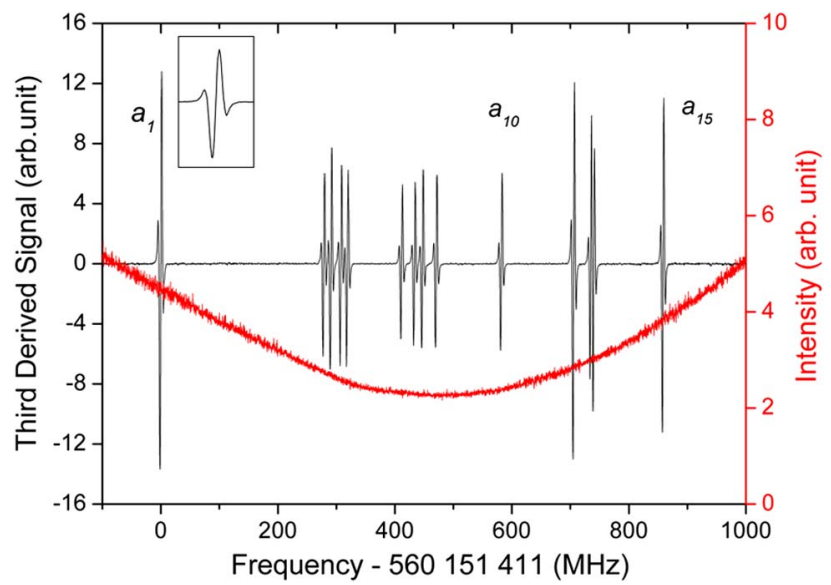

Fig. 2. Hyperfine spectrum $a_{1}-a_{15}$ of the iodine $\mathrm{P}(28) 30-0$ line and the probe beam intensity, which represents the absorption profile. Inset is the signal of $a_{1}$ component, which shows an SNR of 1000 . Here, cold-finger temperature is $14.5^{\circ} \mathrm{C}$ and the lock-in time constant is $30 \mathrm{~ms}$ at $12 \mathrm{~dB} /$ oct. 
the FPI cavity and the laser frequency is scanned across the molecular iodine resonance by tuning the FPI cavity length. The $535 \mathrm{~nm}$ optical powers of the pump and probe beams, both having a diameter of $8.4 \mathrm{~mm}$, are 22.9 and $0.39 \mathrm{~mW}$, respectively. The optical intensity of the probe beam is slightly different for each hyperfine transition due to the iodine absorption. The cold-finger temperature of the iodine cell is held at $14.5^{\circ} \mathrm{C}$ to keep the pressure at $16.5 \mathrm{~Pa}$ inside the cell. The laser frequency is modulated at $13.9 \mathrm{kHz}$ with a modulation width of $5.2 \mathrm{MHz}$ for locking the laser frequency to the hyperfine transition. The well isolated $a_{1}, a_{10}$, and $a_{15}$ hyperfine transitions are chosen for laser stabilization. The inset in Fig. 2 shows the third-derivative signal of the $a_{1}$ line. The SNRs of the $a_{1}, a_{10}$, and $a_{15}$ components at lock-in time constant $30 \mathrm{~ms}$ at $12 \mathrm{~dB} /$ oct are approximately 1000, 500, and 900 , respectively.

The beat frequency between the iodine-stabilized $1070 \mathrm{~nm}$ laser and the OFC is measured using a high-frequency counter (Agilent A53150) with a $100 \mathrm{~ms}$ gate time and 500 data points are recorded for each beat frequency measurement. Then the average value is used to derive the absolute frequency of the hyperfine transition using Eq. (1). To investigate the laser frequency stability, the beat frequency is utilized to calculate the Allan deviation with 1000 data points, shown in Fig. 3. The laser frequency has a fractional Allan deviation of $3 \times 10^{-12}$ at $10 \mathrm{~s}$ averaging time, when the laser was stabilized to the $a_{1}$ component at iodine vapor pressure of $16.5 \mathrm{~Pa}$.

To determine the transition frequency at zero pressure, the pressure shift must be investigated. For pressure shift measurement, the pump and probe powers are fixed at 22.9 and $0.39 \mathrm{~mW}$, respectively, and the cold-finger temperature is changed to control the vapor pressure of the iodine cell. The iodine partial pressure inside the cell is calculated according to the following formula [19]:

$$
\log (P)=\frac{-3512.830}{T}-2.013 \cdot \log (T)+18.37971
$$

where $P$ is the iodine vapor pressure in Pascal and $T$ is the cold-finger temperature in Kelvin. The cold-finger temperature of the iodine cell is varied from $0.1^{\circ} \mathrm{C}(4.2 \mathrm{~Pa})$ to $20.2^{\circ}$ $\mathrm{C}(26.9 \mathrm{~Pa})$. All the measured frequencies in the test pressure range are used to determine the zero-pressure absolute frequency and the pressure shift coefficient. Figure $\underline{4}$ shows

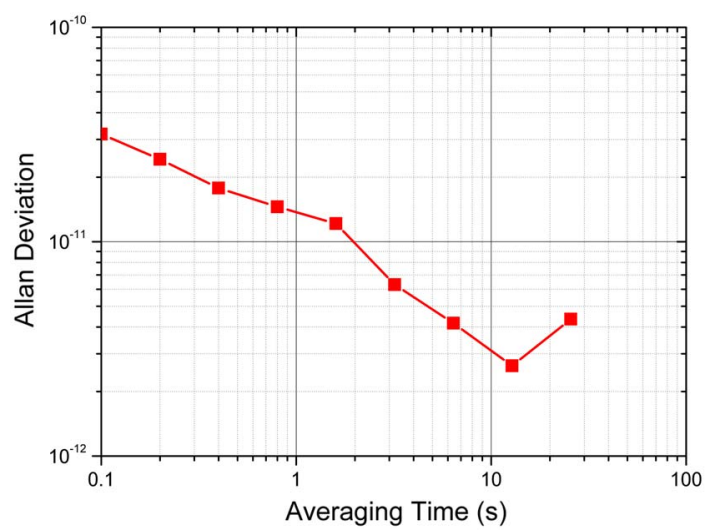

Fig. 3. Fractional Allan deviation of the measured beat frequency between the laser locked on $a_{1}$ component and the OFC.
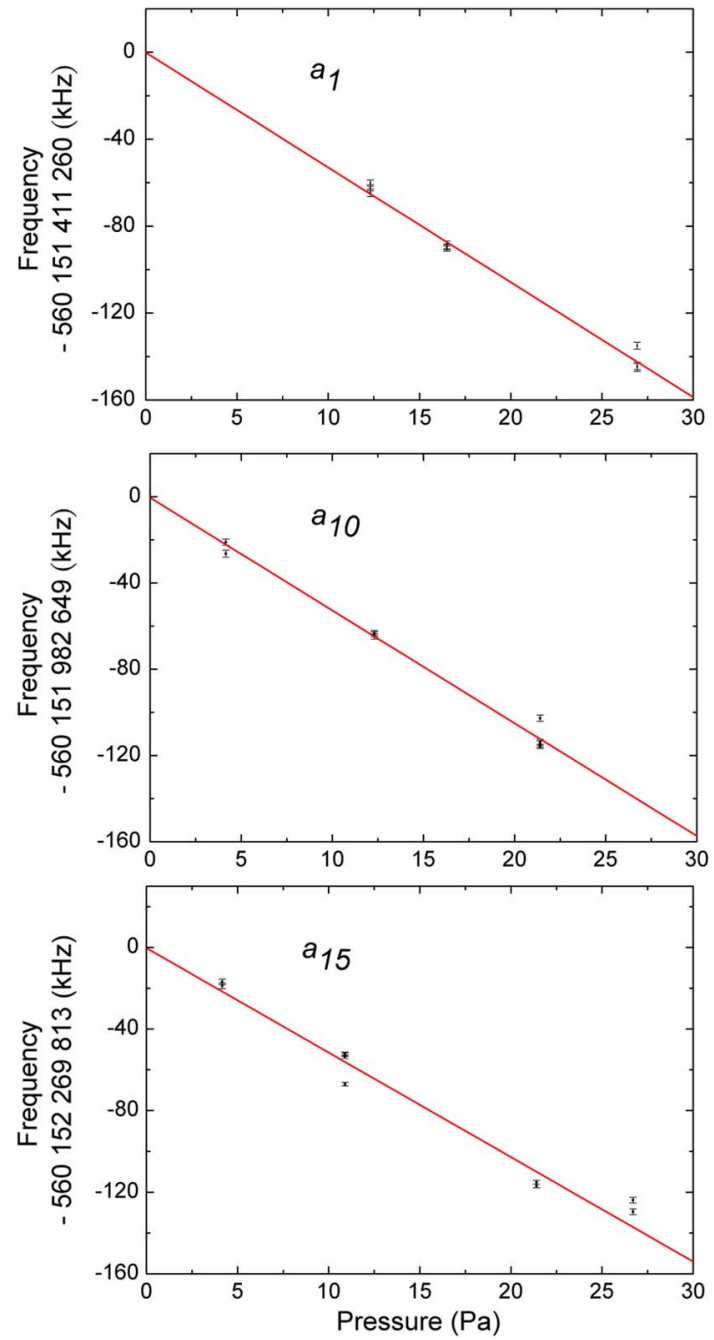

Fig. 4. Measured absolute frequencies versus iodine vapor pressures for hyperfine components $a_{1}, a_{10}$, and $a_{15}$. Each data point represents the mean value of 500 measurements. The standard deviation of the 500 measurements divided by the square root of 500 is assigned as the error bar of each data point.

the frequency measurement versus iodine vapor pressure. The statistical expected zero-pressure absolute frequencies are $560151411260(2) \mathrm{kHz}, 560151982$ 649(1) kHz, and $560152269813(1) \mathrm{kHz}$ for the $a_{1}, a_{10}$, and $a_{15}$ components, respectively. The pressure shift coefficients for the $a_{1}, a_{10}$, and $a_{15}$ components deduced from the linear relationship of the measured absolute frequencies versus vapor pressures are $-5.28 \pm 0.08 \mathrm{kHz} / \mathrm{Pa},-5.23 \pm 0.07 \mathrm{kHz} / \mathrm{Pa}$, and $-5.12 \pm$ $0.05 \mathrm{kHz} / \mathrm{Pa}$ for the $a_{1}, a_{10}$, and $a_{15}$ components.

Besides the correction of the pressure shift, another systematic uncertainty that comes from the servo-loop electronics is found to be around $10 \mathrm{kHz}$. The DC offset from the lock-in amplifier also enters into an uncertainty of around $2 \mathrm{kHz}$. Table 1 shows the measurement results of the three transition frequencies during one month and the comparisons with the calculated values.

To investigate the pressure and power broadening of the $a_{10}$ component, we adopt the method proposed by Fang et al. [20] to determine the linewidth by measuring the dependence of the peak amplitude of the third-derivative signal on the modulation width. The peak amplitude of the third-derivative 
Table 1. Selected Hyperfine Transition Frequencies of the $P(28)$ 30-0 Line and Comparisons to the Calculated Values $(\mathbf{k H z})$

\begin{tabular}{lcccc}
\hline & \multicolumn{2}{c}{ Measured $^{a}$} & Calculated $^{b}$ & Measured-Calculated \\
\hline$a_{1}$ & $560151411260(11)$ & 560151405525 & 5735 \\
$a_{10}$ & $560151982649(11)$ & 560151976906 & 5743 \\
$a_{15}$ & $560152269813(11)$ & 560152264072 & 5741 \\
\hline
\end{tabular}

${ }^{a}$ The uncertainty is the combined error of statistical error and another systematic uncertainties which come from the servo loop and the DC offset of the lock-in amplifier.

${ }^{b}$ Calculated from IodineSpec5 [8].

signal on the normalized modulation width $\delta_{A}$ can be written as

$$
\begin{aligned}
h\left(\delta_{A}\right)= & C \\
& \cdot\left(P_{1} \delta_{A}+P_{2} \delta_{A}^{2}+P_{3} \delta_{A}^{3}\right) /\left(P_{4}+P_{5} \delta_{A}+P_{6} \delta_{A}^{2}+P_{7} \delta_{A}^{3}\right),
\end{aligned}
$$

where $\delta_{A}=d_{A} / d_{h}, d_{A}$ is the modulation amplitude, $d_{h}$ is the half-width at half-maximum of the transition line, and $C$ is a constant. The coefficients are $P_{1}=-1.51636, P_{2}=6.89591$, $P_{3}=-0.09229, P_{4}=48.96763, P_{5}=-3.70996, P_{6}=16.56378$, and $P_{7}=1.93711$. For the pressure broadening measurement, the pump and probe powers are fixed at 22.9 and $0.39 \mathrm{~mW}$, and the cold-finger temperature of the iodine cell is varied from $0.1^{\circ} \mathrm{C}(4.2 \mathrm{~Pa})$ to $20.2^{\circ} \mathrm{C}(26.9 \mathrm{~Pa})$. Figure 5 shows the variation of the linewidth with iodine vapor pressure. The inset in Fig. 5 shows the measured peak amplitude of the third-derivative signal versus the modulation amplitude of the $a_{10}$ component under different vapor pressure. The pressure broadening coefficient is $125(14) \mathrm{kHz} / \mathrm{Pa}$ and the zero-pressure linewidth is 3.72(19) MHz. For the power broadening experiment, shown in Fig. 6, the cold-finger temperature of the iodine cell was fixed at $0.1^{\circ} \mathrm{C}(4.2 \mathrm{~Pa})$, and the pump power was changed from 4.6 to $41.3 \mathrm{~mW}$. The probe power was fixed at $1.7 \%$ of the pump power. The inset in Fig. 6 shows the measured peak amplitude of the third-derivative signal versus the modulation amplitude of the $a_{10}$ component under different pump power. The relation of linewidth and the pump power is [21]

$$
\gamma^{\prime}=\gamma\left(1+\sqrt{1+P / P_{s}}\right)
$$

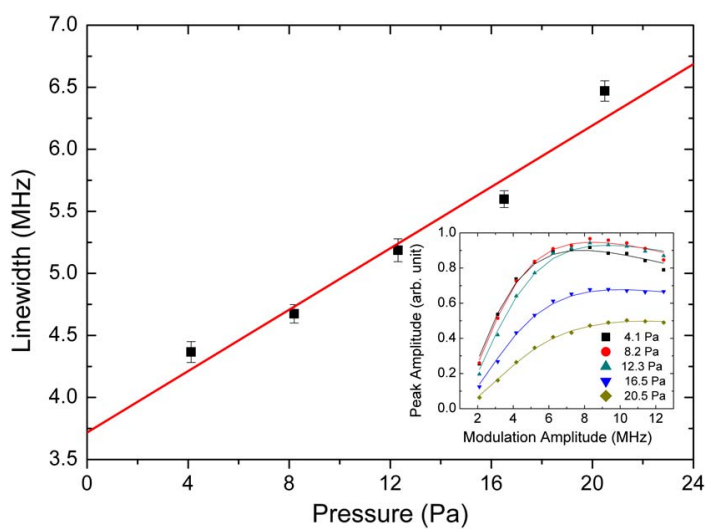

Fig. 5. Linewidth of $a_{10}$ versus the iodine vapor pressure. The pump and probe powers are fixed at 22.9 and $0.39 \mathrm{~mW}$. The inset shows the measured peak amplitude of the third-derivative signal versus the modulation amplitude under different vapor pressure. The solid curves in inset depict results of curve fitting.

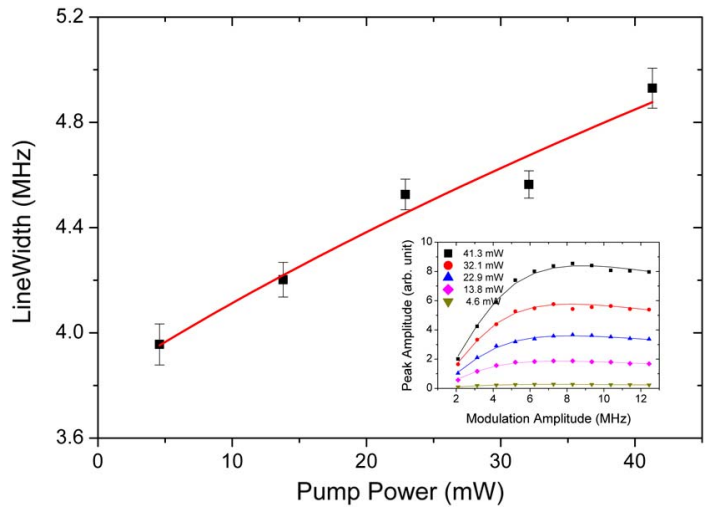

Fig. 6. Linewidth of $a_{10}$ versus pump power. The cold finger temperature of the iodine cell is fixed at $0.1^{\circ} \mathrm{C}(4.2 \mathrm{~Pa})$. The inset shows the measured peak amplitude of the third-derivative signal on the modulation amplitude under different pump power. The solid curves in inset depict results of curve fitting.

where $\gamma$ is the linewidth associated with the limit of weak saturating and probe beams, $P$ is the power in the saturating beam, and $P_{S}$ is the saturation power. Through a nonlinear least-squares fit to the data in Fig. $\underline{6}$, we obtained $\gamma=$ 1.91(4) $\mathrm{MHz}$ and $P_{S}=28.6 \mathrm{~mW}$, corresponding to a saturation intensity of $51.6 \mathrm{~mW} / \mathrm{cm}^{2}$. However, the saturation intensity is not reliable due to the limited available data points. With a linear fit the measured power broadening slope is $12.6(2) \mathrm{kHz} / \mathrm{mW}$.

\section{CONCLUSIONS}

In summary, a frequency-doubled $1070 \mathrm{~nm} \mathrm{Nd:GdVO}$ laser is stabilized to the $a_{1}, a_{10}$, and $a_{15}$ hyperfine transitions of $\mathrm{P}(28)$ 30-0 line of ${ }^{127} \mathrm{I}_{2}$ and their absolute frequencies are measured by an OFC. The pressure shift in the iodine cell is investigated to deduce the zero-pressure absolute frequency. The pressure broadening and power broadening effects are also investigated for the $a_{10}$ hyperfine component. The $\mathrm{P}(28)$ 30-0 line of iodine is close to the $6 \mathrm{P}_{3 / 2}-7 \mathrm{~S}_{1 / 2}$ transition of the thallium atom. This laser system will be employed to investigate the $6 \mathrm{P}_{3 / 2}-7 \mathrm{~S}_{1 / 2}$ transition using a thallium hollow cathode lamp and the PNC effect using the EIT spectrum in atomic thallium.

\section{ACKNOWLEDGMENTS}

This work is supported by the National Science Council and the Ministry of Education of Taiwan, ROC.

\section{REFERENCES}

1. I. Velchev, W. Hogervorst, and W. Ubachs, "Precision VUV spectroscopy of Ar I at 105 nm," J. Phys. B 32, L511-L516 (1999).

2. S. L. Cornish, Y.-W. Liu, I. C. Lane, P. E. G. Baird, G. P. Barwood, P. Taylor, and W. R. C. Rowley, "Interferometric measurements of ${ }^{127} \mathrm{I}_{2}$ reference frequencies for $1 \mathrm{~S}-2 \mathrm{~S}$ spectroscopy in muonium, hydrogen, and deuterium," J. Opt. Soc. Am. B 17, 6-10 (2000).

3. P. Cancio Pastor, G. Giusfredi, P. De Natale, G. Hagel, C. de Mauro, and M. Inguscio, "Absolute frequency measurements of the $2^{3} \mathrm{~S}_{1} \rightarrow 2^{3} \mathrm{P}_{0,12}$ atomic helium transitions around 1083 nm," Phys. Rev. Lett. 92, 023001 (2004).

4. S. Reinhardt, G. Saathoff, H. Buhr, L. A. Carlson, A. Wolf, D. Schwalm, S. Karpuk, C. Novotny, G. Huber, M. Zimmermann, R. Holzwarth, T. Udem, T. W. Hänsch, and G. Gwinner, "Test of relativistic time dilation with fast optical atomic clocks at different velocities," Nat. Phys. 3, 861-864 (2007). 
5. Y.-C. Huang, W.-J. Luo, Y.-T. Kuo, and L.-B. Wang, "Precision measurement of hyperfine intervals in the D1 lines of atomic ${ }^{7}$ Li," J. Phys. B 46, 075004 (2013).

6. T. J. Quinn, "Practical realization of the definition of the metre, including recommended radiations of other optical frequency standards (2001)," Metrologia 40, 103-133 (2003).

7. R. Felder, "Practical realization of the definition of the metre, including recommended radiations of other optical frequency standards (2003)," Metrologia 42, 323-325 (2005).

8. "IodineSpec5," http://www.iqo.uni-hannover.de.

9. A. Arie and R. L. Byer, "Laser heterodyne spectroscopy of ${ }^{127} \mathrm{I}_{2}$ hyperfine structure near $532 \mathrm{~nm}$," J. Opt. Soc. Am. B 10, 1990-1997 (1993).

10. P. A. Jungner, S. Swartz, M. Eickhoff, J. Ye, J. L. Hall, and S. Waltman, "Absolute frequency of the molecular iodine transition R(56)32-0 near 532 nm," IEEE Trans. Instrum. Meas. 44, 151-154 (1995).

11. J. Ye, L. Robertsson, S. Picard, L.-S. Ma, and J. L. Hall, "Absolute frequency atlas of molecular $I_{2}$ lines at $532 \mathrm{~nm}$," IEEE Trans. Instrum. Meas. 48, 544-549 (1999).

12. G. Galzerano, C. Svelto, E. Bava, and F. Bertinetto, "High frequency-stability diode-pumped Nd:YAG lasers with the FM sidebands method and Doppler-free iodine lines at $532 \mathrm{~nm}$," Appl. Opt. 38, 6962-6966 (1999).

13. F.-L. Hong and J. Ishikawa, "Hyperfine structures of the R (122) 35-0 and $\mathrm{P}(84) 33-0$ transitions of ${ }^{127} \mathrm{I}_{2}$ near $532 \mathrm{~nm}$," Opt. Commun. 183, 101-108 (2000).
14. N. H. Edwards, S. J. Phipp, P. E. G. Baird, and S. Nakayama, "Precise measurement of parity nonconserving optical rotation in atomic thallium," Phys. Rev. Lett. 74, 2654-2657 (1995).

15. P. A. Vetter, D. M. Meekhof, P. K. Majumder, S. K. Lamoreaux, and E. N. Fortson, "Precise test of electroweak theory from a new measurement of parity nonconservation in atomic thallium," Phys. Rev. Lett. 74, 2658-2661 (1995).

16. A. D. Cronin, R. B. Warrington, S. K. Lamoreaux, and E. N. Fortson, "Studies of electromagnetically induced transparency in thallium vapor and possible utility for measuring atomic parity nonconservation," Phys. Rev. Lett. 80, 3719-3722 (1998).

17. N.-C. Shie, W.-F. Hsieh, and J.-T. Shy, "Single frequency $1070 \mathrm{~nm}$ $\mathrm{Nd}: \mathrm{GdVO}_{4}$ laser using a volume Bragg grating," Opt. Express 19 21109-21115 (2011).

18. C.-C. Liao, K.-Y. Wu, Y.-H. Lien, H. Knöckel, H.-C. Chui, E. Tiemann, and J.-T. Shy, "Precise frequency measurements of ${ }^{127} \mathrm{I}_{2}$ lines in the wavelength region $750-780 \mathrm{~nm}$," J. Opt. Soc. Am. B 27, 1208-1214 (2010).

19. L. J. Gillespie and L. H. D. Fraser, "The normal vapor pressure of crystalline iodine," J. Am. Chem. Soc. 58, 2260-2263 (1936).

20. H.-M. Fang, S. C. Wang, and J.-T. Shy, "Pressure and power broadening of the $a_{10}$ component of R(56)32-0 transition of molecular iodine at 532 nm," Opt. Commun. 257, 76-83 (2006).

21. V. S. Letokhov and V. P. Chebotayev, "Elements of theory of resonant interaction of a laser field and gas," in Nonlinear Laser Spectroscopy (Springer, 1977), pp. 72-80. 